ARTICLE

\title{
Neutron-Coupled Thermal Hydraulic Calculation of BWR under Seismic Acceleration
}

\author{
Akira SATOU*, Tadashi WATANABE, Yu MARUYAMA and Hideo NAKAMURA \\ Japan Atomic Energy Agency, 2-4 Shirakata, Tokai-mura, Naka-gun, Ibaraki-ken, 319-1195, Japan
}

\begin{abstract}
In the BWR subjected to an earthquake, the oscillating acceleration attribute to the seismic wave may cause the variation of the coolant flow rate and void fraction in the core, which might result in the core instability due to the void-reactivity feedback. Therefore, it is important to properly evaluate the effect of the seismic acceleration on the core stability from a viewpoint of plant integrity estimation. In the present study the numerical code analyzing the behavior of nuclear power plant under the seismic acceleration is developed based on the three-dimensional neutron-coupled thermal hydraulic code, TRAC-BF1/SKETCH-INS. The coolant flow in the core is simulated with introducing the oscillating acceleration attributed to the earthquake motion into the motion equation of the two-phase flow as external force terms. The analyses are performed on a real BWR4-type nuclear power plant (Peach Bottom \#2) with the sinusoidal acceleration and the acceleration obtained from the response analysis to the El Centro seismic wave of Imperial Valley earthquake. The behaviors of the core and coolant are calculated in the various parameters of acceleration. The effects of the frequency, amplitude and direction of the oscillating acceleration are discussed.
\end{abstract}

KEYWORDS: earthquake, neutronics/thermal-hydraulics coupling, BWR stability

\section{Introduction}

When a nuclear power plant is subjected to a huge earthquake, the seismic vibration propagates through the core internals and shakes the coolant water with measurable oscillating acceleration compared with the gravitational one. So far, the effects of seismic vibration on a nuclear power plant have been researched mainly on the viewpoint of the integrity of structure or equipments, whereas only a few researches concerning the effects of the vibration on coolant flow has been performed. In a boiling water reactor (BWR), the coolant flow rate and void fraction will fluctuate under the oscillating acceleration. It will cause the fluctuation of the core power through the void-reactivity feedback and induce the core instability. Hirano et al. have modified the best estimate code, TRAC-BF1, to take into account the external acceleration and investigated the BWR instability due to seismically induced resonance within the scope of a point kinetics model. ${ }^{1)}$ They have displayed that the instability was caused by the resonance between the oscillating acceleration and the core-wide oscillation, of which period was about 5 seconds, due to the nuclear-coupled thermal hydraulic instabilities. It has been clarified that the long-period and large-amplitude oscillation beyond the previous expectation will continue for a long term if the massive earthquake anticipated in Japan today occurs. It is important to evaluate the core instability under such oscillating condition from the view point of plant integrity.

In the present study, the numerical code is developed based on the three-dimensional neutron-coupled thermal

*Corresponding author, E-mail:satou.akira@jaea.go.jp

(c) 2011 Atomic Energy Society of Japan, All Rights Reserved. hydraulic code, TRAC-BF1/SKETCH-INS to calculate the nuclear plant behavior under seismic oscillation. The analyses are performed on a real BWR4-type nuclear power plant with the sinusoidal acceleration and the acceleration obtained from the response analysis to a real earthquake wave. The behaviors of the core and coolant are calculated in the various parameters of acceleration. The effects of the frequency, amplitude and direction of the oscillating acceleration are discussed.

\section{Methods and Models}

\section{Code Overview}

The TRAC-BF1 developed by the US NRC is the best-estimate two-fluid model thermal-hydraulic code, where the mass, momentum and energy equations are solved for both gas and liquid phases. ${ }^{2}$ This code also includes 1-dimensional neutron kinetics model for nuclear calculation, however, the 1-D kinetics models significantly limit the best-estimate capabilities of the code. To remove this limitation a coupling with a 3D neutron kinetics code SKETCH-INS has been performed. The SKETCH-INS, refined version of the SKETCH-N code, ${ }^{3)}$ solves neutron diffusion equations in $\mathrm{X}-\mathrm{Y}-\mathrm{Z}$ geometry for steady-state and neutron kinetics problems. The code can treat an arbitrary number of neutron energy groups and delayed neutron precursors. TRAC-BF1/SKETCH-INS code is assembled by coupling between the TRAC and SKETCH codes using the interface module based on the message-passing library $\mathrm{Pa}$ rallel Virtual Machine (PVM). The interface module is responsible for data transfer between the codes, mapping of the data between different spatial meshes and synchronization of the time stepping. 


\section{Code Modification}

Assuming that the plant oscillates as a whole like a rigid body, we can introduce the external acceleration term by adding to the gravitational acceleration term in the motion equation. In TRAC-BF1, an analysis model is composed of 3-dimensional components (e.g. pressure vessel) and 1-dimensional components (e.g. fuel bundles and piping). For the 3-dimensional components, the external acceleration vector is directly added to the gravitational acceleration vector. For the 1-dimensional components, the coordinate transformation of the acceleration and the modification of a pressure term attributed to the difference of void fraction between horizontally adjacent cells are needed. The differential form of the motion equation for the 1-dimensional components in TRAC-BF1 can be written for the gas motion,

$$
\begin{aligned}
& \frac{V_{g}^{n+1}-V_{g}^{n}}{\Delta t}+V_{g} \nabla_{j+1 / 2} V_{g}+k_{v m} \frac{\rho_{c, j+1 / 2}}{\left(\overline{\alpha \rho_{g}}\right)_{j+1 / 2}^{n}} \frac{\left(V_{g}^{n+1}-V_{l}^{n+1}\right)-\left(V_{g}^{n}-V_{l}^{n}\right)}{\Delta t} \\
& +\frac{C_{i}^{n}\left|V_{g}^{n}-V_{l}^{n}\right|\left(V_{g}^{n+1}-V_{l}^{n+1}\right)}{\left(\overline{\alpha \rho_{g}}\right)_{j+1 / 2}^{n}}+\frac{P_{j+1}^{n+1}-P_{j}^{n}}{\left(\overline{\rho_{g}}\right)_{j+1 / 2}^{n} \cdot \overline{\Delta x}}+\frac{C_{w g}\left|V_{g}^{n}\right| V_{g}^{n+1}}{\left(\overline{\alpha \rho_{g}}\right)_{j+1 / 2}^{n}}+g \cos \theta \\
& +k_{v m} \frac{\rho_{c, j+1 / 2}^{n}}{\left(\overline{\alpha \rho_{g}}\right)_{j+1 / 2}^{n}} V_{D}^{n} \nabla_{j+1 / 2}\left(V_{g}-V_{l}\right)=0
\end{aligned}
$$

and for the liquid motion,

$$
\begin{aligned}
& \frac{V_{l}^{n+1}-V_{l}^{n}}{\Delta t}+V_{l} \nabla_{j+1 / 2} V_{l}+k_{v m} \frac{\rho_{c, j+1 / 2}}{\left(\overline{(1-\alpha) \rho_{l}}\right)_{j+1 / 2}^{n}} \frac{\left(V_{l}^{n+1}-V_{g}^{n+1}\right)-\left(V_{l}^{n}-V_{g}^{n}\right)}{\Delta t} \\
& +\frac{C_{i}^{n} \mid V_{g}^{n}-V_{l}^{n}\left(V_{l}^{n+1}-V_{g}^{n+1}\right)}{\left(\overline{(1-\alpha) \rho_{l}}\right)_{j+1 / 2}^{n}}+\frac{P_{j+1}^{n+1}-P_{j}^{n}}{\left(\overline{\rho_{l}}\right)_{j+1 / 2}^{n} \cdot \overline{\Delta x_{j+1 / 2}}}+\frac{C_{w l}\left|V_{l}^{n}\right| V_{l}^{n+1}}{\left(\overline{(1-\alpha) \rho_{l}}\right)_{j+1 / 2}^{n}}+g \cos \theta \\
& +k_{v m} \frac{\rho_{c, j+1 / 2}^{n}}{\left(\overline{(1-\alpha) \rho_{l}}\right)_{j+1 / 2}^{n}} V_{D}^{n} \nabla_{j+1 / 2}\left(V_{l}-V_{g}\right)+\frac{\nabla P_{s}^{n}}{\left(\overline{(1-\alpha) \rho_{l}}\right)_{j+1 / 2}^{n}}=0
\end{aligned}
$$

where $g$ and $l$ denote the gas and liquid phases respectively, $n$ is the time step, $j$ is the cell number, $V$ is velocity, $k_{v m}$ is the virtual mass coefficient, $C_{i}$ is the interfacial shear coefficient, $C_{w}$ is the wall shear coefficient, $\rho$ is the density, $P$ is the pressure, $\Delta t$ is the time difference, $\alpha$ is the void fraction, $\Delta x$ is the distance between adjacent cells, $\theta$ is the angle of $\mathrm{z}$-axis in a vertical plane, and $g \cos \theta$ is the component of gravity in the flow direction. A component of the external acceleration vector $\vec{a}=\left(a_{x}, a_{y}, a_{z}\right)$ in the flow direction is written as

$$
\vec{n} \cdot \vec{a}=a_{z} \cos \theta+\left(a_{x} \cos \phi+a_{y} \sin \phi\right) \sin \theta
$$

where, $\vec{n}$ is the unit vector in the flow direction expressed by

$$
\vec{n}=(\sin \theta \cos \phi, \sin \theta \sin \phi, \cos \theta)
$$

and $\phi$ is the angle of $\mathrm{x}$-axis in a horizontal plane. Subtracting Eq. (3) from the left-hand sides of Eqs. (2) and (3), the external acceleration as a body force can be introduced. ${ }^{1)}$

In addition, the pressure difference in Eq. (2), $\nabla P_{s}$, attri-

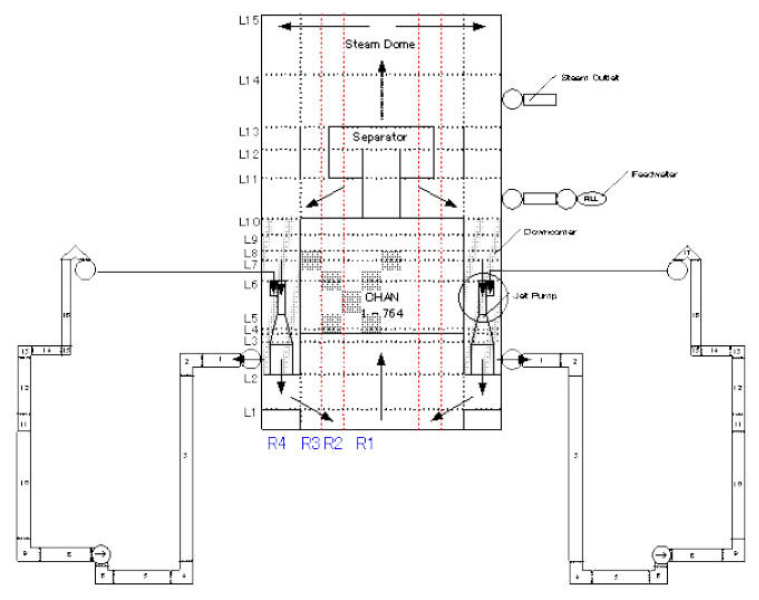

(a) Plant nodalization

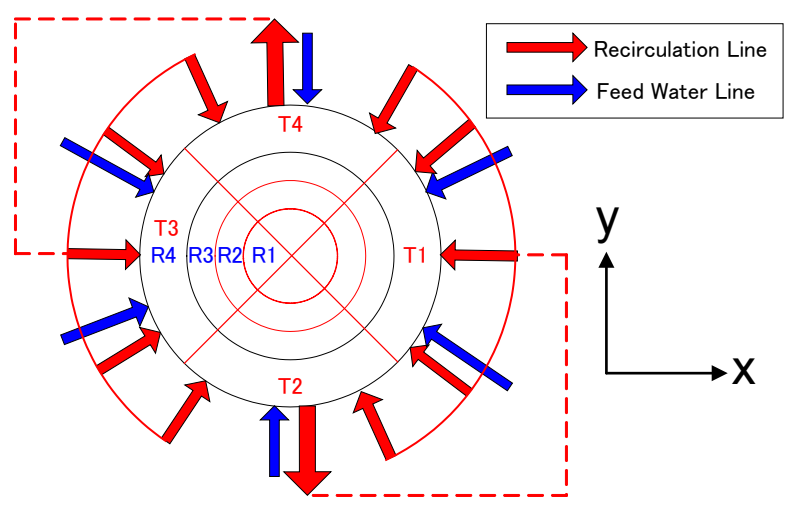

(b) vessel noding and alignment of piping installation

Fig. 1 Plant modelling

buted to the difference of void fraction between horizontally adjacent cells written in following,

$$
\nabla P_{s}=\left(\rho_{l}-\rho_{g}\right) g \Delta Z\left(\alpha_{2}-\alpha_{1}\right) \sin \theta
$$

is affected by the horizontal component of the external acceleration. Therefore, Eq. (5) is substituted by following equation,

$$
\nabla P_{s}=\left(\rho_{l}-\rho_{g}\right)\left(g-a_{z}\right) \Delta Z\left(\alpha_{2}-\alpha_{1}\right) \sin \theta
$$

\section{Plant Modeling}

The analyses are performed on a real BWR4-type nuclear power plant (Peach Bottom \#2) consists of two recirculation loops, twenty jet pumps and 764 fuel bundles. Plant geometry, pipe alignment, operating condition and so forth were modeled by using reference from the OECD's turbine trip benchmark. ${ }^{4)}$ In the present study the plant was modeled by 1-dimensional components, whereas the pressure vessel was modeled by 3-dimensional components as shown in Fig. 1(a). The vessel was nodalized into 4 circumferential sections, 4 radial sections, and 15 axial sections. Six inlets of the feed water line and two recirculation loops with 2 outlets and 10 inlets were modeled as shown in Fig. 1(b). All the fuel bundles were modeled individually by CHAN components axially nodalized into 27 cells. So far only a full power 
(3293 MW) operation condition without scrum and pump trip was taken into consideration.

\section{Results and Discussion}

\section{Sinusoidal External Acceleration}

Calculations were performed under sinusoidal external acceleration to avoid a complexity. The acceleration can be written as

$$
a_{x}=a_{x}^{*} \sin \left(\frac{2 \pi}{\tau} t\right), \quad a_{y}=a_{y}^{*} \sin \left(\frac{2 \pi}{\tau} t\right), \quad a_{z}=a_{z}^{*} \sin \left(\frac{2 \pi}{\tau} t\right)
$$

where, $\mathrm{a}_{\mathrm{x}}{ }^{*}, \mathrm{a}_{\mathrm{y}}{ }^{*}, \mathrm{a}_{\mathrm{z}}{ }^{*}$ and $\tau$ were varied as Table 1.

Table 1 Condition of applied acceleration

\begin{tabular}{|c|c|c|c|c|}
\hline & $\mathrm{a}_{\mathrm{x}}{ }^{*}\left(\mathrm{~m} / \mathrm{s}^{2}\right)$ & $\mathrm{a}_{\mathrm{y}}{ }^{*}\left(\mathrm{~m} / \mathrm{s}^{2}\right)$ & $\mathrm{a}_{\mathrm{z}}{ }^{*}\left(\mathrm{~m} / \mathrm{s}^{2}\right)$ & $\tau(\mathrm{s})$ \\
\hline Case 1 & 0.0 & 0.0 & 9.8 & 2.0 \\
Case 2 & 0.0 & 9.8 & 0.0 & 2.0 \\
Case 3 & 9.8 & 0.0 & 0.0 & 2.0 \\
Case 4 & 9.8 & 9.8 & 9.8 & 2.0 \\
Case 5 & 9.8 & 9.8 & 9.8 & 1.0 \\
Case 6 & 4.9 & 4.9 & 4.9 & 2.0 \\
\hline
\end{tabular}

Cases 1 to 4 were performed to investigate the effects of the acceleration direction. Cases 4 to 6 were to investigate the effects of the oscillation period and amplitude.

(1) Effects of Acceleration Direction

Figure 2 shows the time variation of the cell power distribution in Case 1, i.e., only the vertical acceleration was applied. The vertical location is the 14th cell from the bottom of the channel, that is, the center of the core length. The horizontal location is shown by a line in the schematic of the core cross section in the figure. The cell power oscillated as a whole with the period of about 2 seconds equal to the acceleration period and kept the initial distribution profile through the analysis duration. According to the result, the vertical acceleration causes the power oscillation all over the core in a same phase. Therefore, if the frequency of the vertical acceleration approaches that of core-wide instability, the amplitude of power would increase with time by resonance.

Figure 3 shows the time variation of the cell power distribution in Case 3, i.e., only the horizontal (x-direction) acceleration was applied. The vertical location is the same as Case 1 and the horizontal location is on the core center line in the $\mathrm{x}$-direction. The amplitude of the variation is smaller than that of Case 1, therefore, the effect of the horizontal acceleration on the core power is weaker than that of the vertical acceleration. The period of cell power oscillation was about 2 seconds, however, the phase is different depending on the location. Figure 4(a) and (b) shows the cell power and void fraction variation respectively at the both ends ( $x \_1$ and $\left.x \_30\right)$ and center of the core (x_15). The phase of the cell power variation at the both ends is almost opposite each other. The horizontal acceleration causes the horizontal distribution of flow rate at core inlet, and then this distribution induces the difference of void fraction among the channels as shown in Fig. 4(b). It causes the phase lag of

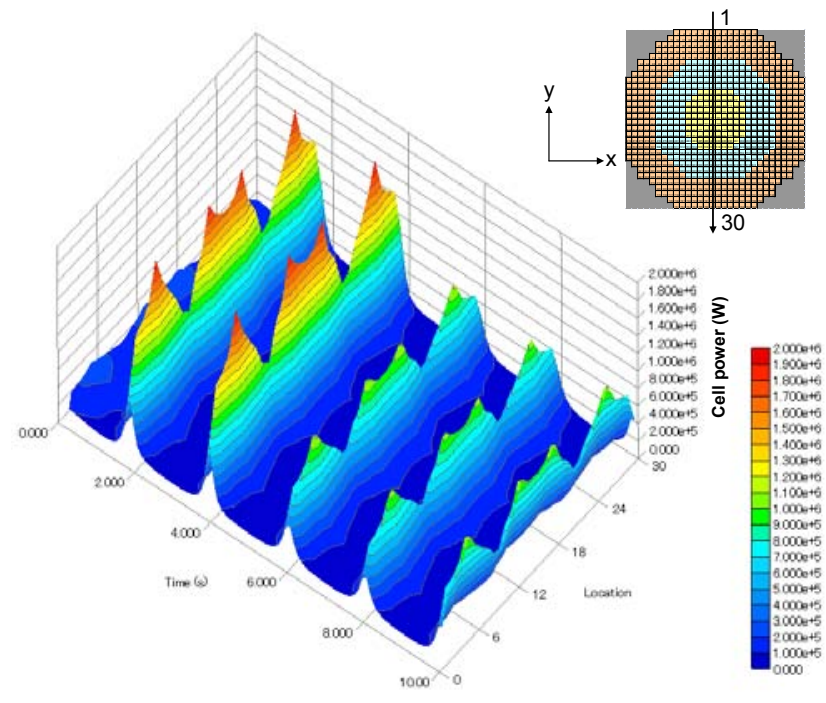

Fig. 2 Time variation of cell power (Case 1)

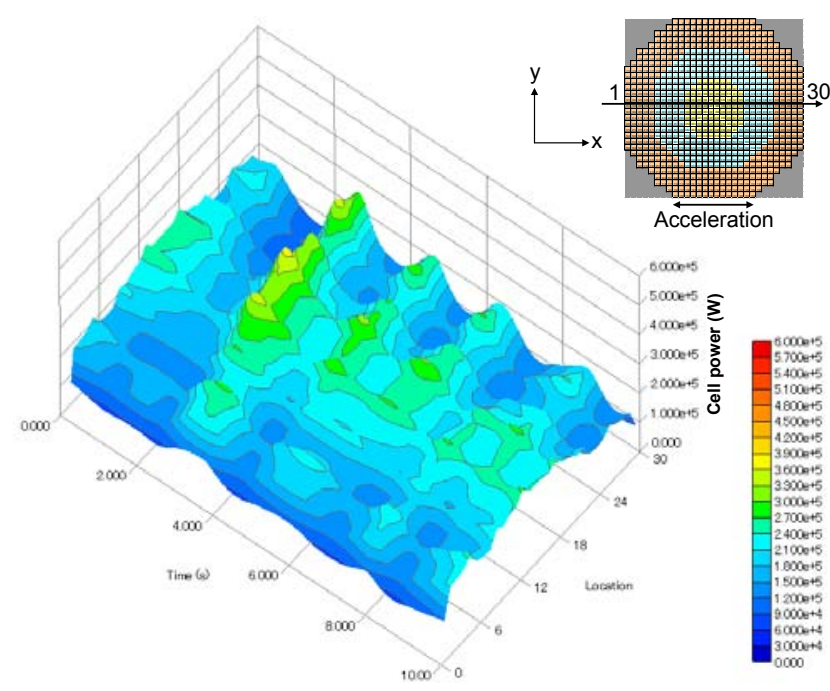

Fig. 3 Time variation of cell power (Case 3)

the cell power through the void-reactivity feedback. From the result, the horizontal acceleration causes the out-of-phase oscillation of the cell power. Therefore, the resonance between regional instability and acceleration would occur if the frequency of them approaches each other.

Figure 5 shows the time variation of the cell power distribution in Case 4. The horizontal location is on the $\mathrm{x}$-direction center line. As the general profile of the variation is analogous with that of Case 1, the effect of the vertical acceleration is clearly strong. However, some difference can be seen. The first peek around 2 second is obviously smaller and the amplitude of the second peek is larger than those of Case 1. And the initial distribution profile is not kept. These would be caused by the nonlinear interaction between the effects of the vertical and horizontal acceleration.

Figure 6 shows the core total power of Cases 1 to 4 . It is displayed that the amplitude of power depends strongly on 


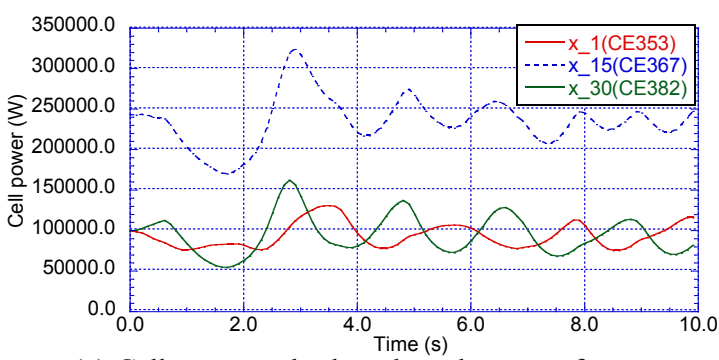

(a) Cell power at both ends and center of core

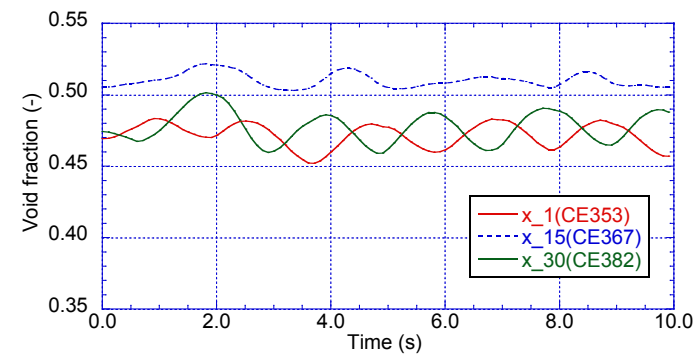

(b) Void fraction at both ends and center of core

Fig. 4 Cell power and void fraction at both ends and center of core

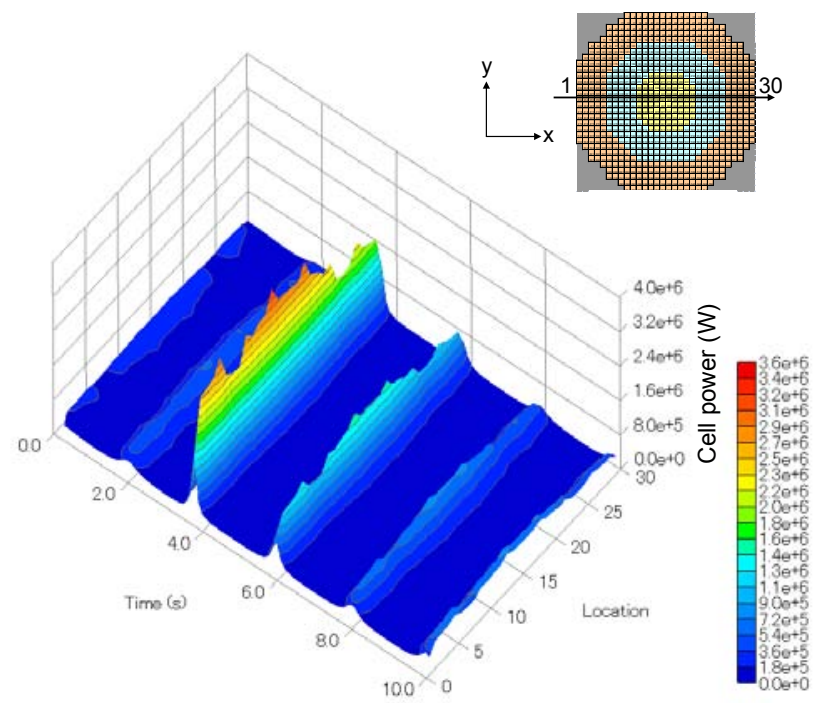

Fig. 5 Time variation of cell power (Case 4)

the vertical acceleration. The effect of the horizontal acceleration is relatively weak, however, it causes the nonlinear interaction with the vertical effect according to the result of Case 4.

(2) Effects of Amplitude and Period of Acceleration

Figure 7 shows the effects of amplitude and period of the acceleration on the total core power. Comparing Case 4 with Case 5, the power response to the acceleration drastically changes depending on the period. When $\tau=1 \mathrm{sec}$, the profile of power variation is sinusoidal and the amplitude is relatively small. When $\tau=2 \mathrm{sec}$, the power response is nonlinear, in which the amplitude is different at every peek and is significantly large at the second peek. From the results of Case 4 and Case 6, the amplitude of the acceleration mainly affects the amplitude of the power and has less in-

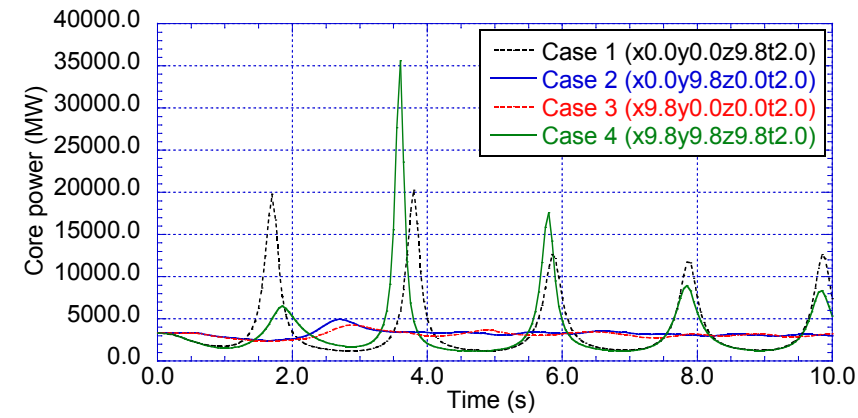

Fig. 6 Total core power

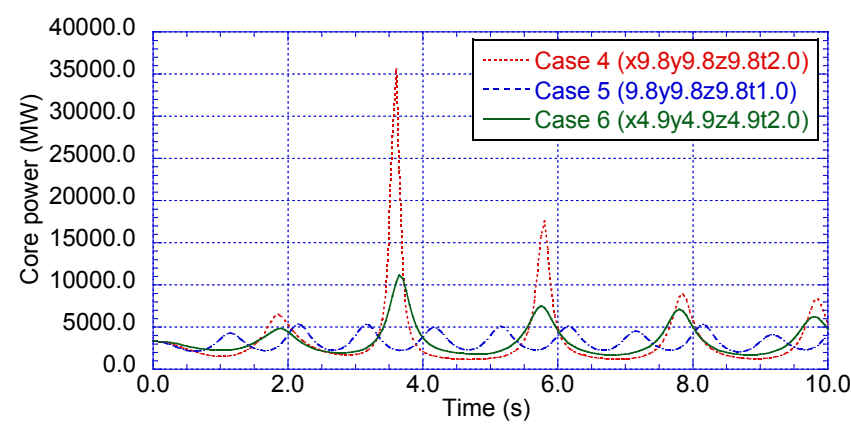

Fig. 7 Total core power

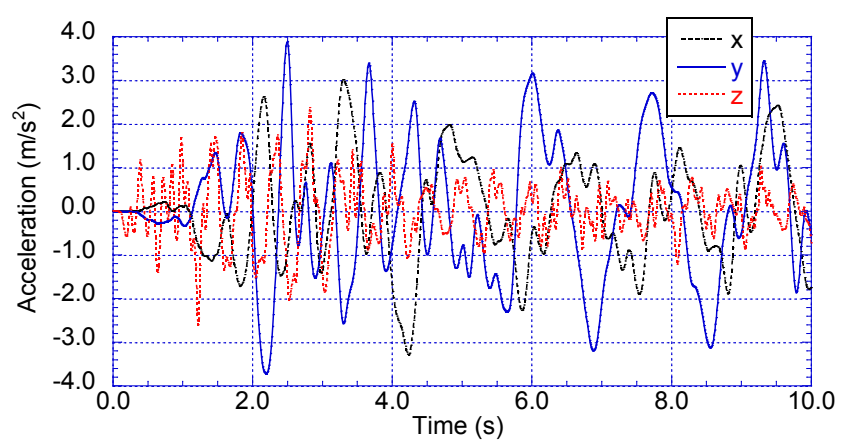

Fig. 8 Time variation of acceleration (Case 7)

fluence on the profile of power variation.

\section{Actual Seismic Acceleration}

Sensitivity analyses were performed by using the oscillating acceleration obtained by the response analysis of the bottom part of a pressure vessel to the El Centro wave of Imperial Valley earthquake, which is the original wave shown in Fig. 8. Variation of the amplitude and period are shown in Table 2. For example, in Case 11, the amplitude of the wave was doubled in any direction and the wave shape was stretched double to the time direction.

Figure 9 shows the time variation of the total core power for every case. The longer the period increased, the larger the amplitude of core power became. Comparing Case 7 and Case 10, the power amplitude of Case 10 was slightly larger than that of Case 7. According to the result, the period of acceleration significantly influenced on the core power. Therefore, it is important to evaluate the effect of the earthquake with long-period oscillating acceleration on the core stability. 
Table 2 Multiplication factor for acceleration

\begin{tabular}{|c|c|c|c|c|}
\hline & \multicolumn{4}{|c|}{ Multiplication factor } \\
\cline { 2 - 5 } & $\mathrm{x}$ & $\mathrm{y}$ & $\mathrm{z}$ & $\tau$ \\
\hline $\begin{array}{c}\text { Case 7 } \\
\text { (Original wave) }\end{array}$ & 1 & 1 & 1 & 1 \\
\hline Case 8 & 1 & 1 & 1 & 2 \\
\hline Case 9 & 1 & 1 & 1 & 4 \\
\hline Case 10 & 2 & 2 & 2 & 1 \\
\hline Case 11 & 2 & 2 & 2 & 2 \\
\hline
\end{tabular}

\section{Conclusion}

The analysis code was developed based on the 3-dimensional neutron-coupled thermal hydraulic analysis code, TRAC-BF1/SKETCH-INS, in order to calculate the nuclear plant behavior under seismic oscillation.

Through the analyses with the 3-dimensional neutron kinetics and the full channel core modeling, the external acceleration had the capability to cause the resonance with the both core-wide and regional instability. Especially the vertical acceleration strongly influence on the core power oscillation. Although the horizontal acceleration alone caused the small power fluctuation, it caused the large effect by the nonlinear interaction with the effect of the vertical acceleration.

Through the parametric calculations with varying the amplitude and period of the acceleration, the period had a significant influence on the core power oscillation. It indicates that the evaluation of the effect of the earthquake with long-period oscillation on the core stability is necessary to keep the integrity of the nuclear power plant.

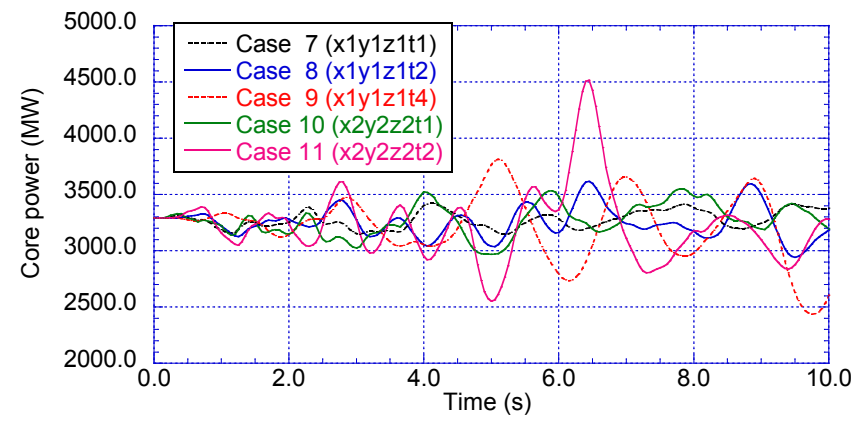

Fig. 9 Total core power

\section{Acknowledgment}

This research has been supported by the Japan Science and Technology Agency Grant (CREST: Core Research for Evolutional Science and Technology).

\section{References}

1) M. Hirano, T. Tamakoshi, "An analytical study on excitation of nuclear-coupled thermal hydraulic instability due to seismically induced resonance in BWR," Nucl. Eng. Des., 162, 307-315 (1996).

2) W. L. Weaver et al., TRAC-BF1 manual: extensions to TRAC-BD1/MOD1, NUREG/CR-4391, EGG-2417, (1986).

3) V. G. Zimin, H. Ninokata, "Nodal neutron kinetics model based on nonlinear iteration procedure for LWR analysis," Ann. Nucl. Energy, 25, 507-528 (1998).

4) J. Solis et al., Boiling water reactor turbine trip (TT) benchmark (Volume I: Final specifications), NEA/NSC/DOC(2001)1, (2001). 